\title{
Interactive Multisensory Environments for Primary School Children
}

\author{
Franca Garzotto \\ Dept. of Electronics, \\ Information and Bio- \\ engineering \\ Politecnico di Milano \\ Milan, Italy \\ franca.garzotto@polimi.it
}

\author{
Eleonora Beccaluva \\ Dept. of Electronics, \\ Information and Bio- \\ engineering \\ Politecnico di Milano \\ Milan, Italy \\ eleonora.beccaluva@polimi.it
}

\author{
Mattia Gianotti \\ Dept. of Electronics, \\ Information and Bio- \\ engineering \\ Politecnico di Milano \\ Milan, Italy \\ mattia.gianotti@polimi.it
}

\author{
Fabiano Riccardi \\ Dept. of Electronics, \\ Information and Bio- \\ engineering \\ Politecnico di Milano \\ Milan, Italy \\ fabiano.riccardi@polimi.it
}

\begin{abstract}
Interactive Multi-Sensory Environments (iMSEs) are roomsized interactive installations equipped with digitally enriched physical materials and ambient embedded devices. These items can sense users' presence, gestures, movements, and manipulation, and react by providing gentle stimulation (e.g., light, sound, projections, blowing bubbles, tactile feel, aromas) to different senses. Most of prior research on iMSEs investigates their use for persons with disabilities (e.g., autism). Our work focuses on the use of iMSEs in primary education contexts and for mixed groups of young students, i.e., children with and without disability. The paper describes the latest version of an iMSE called Magic Room that has been installed in two local schools. We report two empirical studies devoted to understand how the Magic Room could be used in inclusive educational settings, and to explore its potential benefits.
\end{abstract}

\section{Author Keywords}

Interactive Multisensory Environment; Children; Children with Special Needs; Primary School Education; Well-being; Smart Object; Smart Space; Embodied Interaction

\section{CSS Concepts}

- Human-centered computing Empirical studies in ubiquitous and mobile computing • Human-centered computing Empirical studies in $\mathrm{HCI} \bullet$ Social and professional topics $\sim$ People with disabilities, Children • Computer systems organization $\sim$ Sensors and actuators

\section{INTRODUCTION}

A Multisensory Environment (MSE) is a dedicated indoor space that provides a variety of sensory experiences to promote motivation, interests, leisure, and relaxation. The most common use of MSEs is to facilitate the achievement

\footnotetext{
Permission to make digital or hard copies of all or part of this work for personal or classroom use is granted without fee provided that copies are not made or distributed for profit or commercial advantage and that copies bear this notice and the full citation on the first page. Copyrights for components of this work owned by others than ACM must be honored. Abstracting with credit is permitted. To copy otherwise, or republish, to control on servers or to redistribute to lists, requires prior specific permission and/or a fee. Request permissions from Permissions@ acm.org.

CHI 2020, April 25-30, 2020, Honolulu, HI, USA.

(C) 2020 Copyright is held by the owner/author(s). Publication rights licensed to ACM. ACM ISBN 978-1-4503-6708-0/20/04...\$15.00.

DOI: https://doi.org/10.1145/3313831.3376343
}

of specific therapeutic or educational goals among people with severe cognitive or motor impairments. A typical MSE includes lights, visual contents on screens or projections, blowing bubbles, wall panels, objects or furniture that provide focused tactile feelings, auditory stimuli such as songs, music, or nature environment sounds, and flavored aromas. Stimuli are manually controlled (activated, intensified, or reduced) by the caregiver or are generated by effect of user manipulation (e.g., using switches on objects) to promote a sense of agency and cause-effect understanding.

Advances in cyber-physical systems have enabled the transition from traditional to digitally-enhanced MSEs (hereinafter interactive MSEs, or iMSEs). These are roomsized installations equipped with a network of connected digital devices embedded into physical objects, materials, furniture, walls, or floor and typically integrated with immersive projections. Thanks to these technologies, the physical items and the entire space can sense, and react to, the users' presence and their gestures, movements, and manipulation, orchestrating the multisensory stimuli in a countless number of ways.

Limited HCI research exists on iMSEs and in most cases these installations have been designed for users with disability, e.g., children with autism [19][20][21]. According to the existing literature, the empirical studies to evaluate their benefits were performed in laboratory contexts, therapeutic centers, exhibitions or museums, but such researches have not led to a subsequent adoption of the systems in real settings. This paper describes the latest version of an iMSE called Magic Room that has been installed in two local primary schools, has been evaluated with mixed groups of children - with and without disabilityand, at the time of writing this paper, is used on a regular base. The main contribution of our work is to offer insights on how iMSEs can be exploited in primary education contexts to provide inclusive environments for group experiences involving children with different abilities. 


\section{RELATED WORK}

\section{Theoretical Background}

The multisensory approach of both traditional MSEs and interactive MSEs is grounded on the principles of embodied cognition and sensory integration theories. Embodied cognition posits that learning is both an intellectual and a physical process, and emphasizes the formative role of embodiment (the way an organism's sensorimotor capacities enable it to interact with the physical environment successfully) in the development of cognitive skills, such as mental imagery, memory, reasoning and problem solving [35][12]. Sensory integration theories hypothesize that the learning processes and the ability to plan and organize appropriate behaviors involve the ability to assimilate, integrate, and manage sensory information of different natures simultaneously. As a consequence, impairments in the capability of processing stimuli affect many fundamental cognitive and functional abilities and lead to limited interest, attention deficits, inadequate self-regulation [2], difficulty to filter distractions (e.g., background noise or bright lights [32]). These impairments are common for example among people with ASD - Autism Spectrum Disorder, and more broadly persons with NDD - Neurodevelopmental Disorders. Most of specific interventions for this population aim at stimulating the basic sensory mechanisms and promoting "perceptual learning" [6][35]; in some cases, various forms of interactive technologies are used ranging from simple standard devices such as tablets or PCs ([9][34]) to more sophisticated solutions such as iMSE Interactive MultiSensory Environments.

\section{(Interactive) Multi-Sensory Environments}

The ancestors of Interactive Multi-Sensory Environments (iMSEs) are low-tech Multi-Sensory Environments [20]. The most widely known of them is Snoezelen [15], a commercial product which dates back to 30 years ago. Although the empirical evidence of its therapeutic effectiveness is very limited, Snoezelen has been installed in many special education schools and therapeutic centers (mainly in US, UK, and Australia) to offer a relaxing space for persons with severe cognitive impairments to mitigate anxiety, stimulate engagement, and encourage communication with caregivers.

One of the first examples of iMSE is MEDIATE [23][24], designed as a free play multisensory space for children with ASD. MEDIATE includes a large display colored with dynamic digital tassels that change size according to the child's distance, soft and wraparound materials, a multimaterial tube to feel different tactile effects, and a sound floor that generates different sounds according the child's position. The system is equipped with a self-tuning system that senses stereotyped behaviors and automatically decreases the intensity of the stimuli when the same behavioral pattern is repeatedly recognized, to stimulate the child to try other patterns of interaction.

Lands of Fog [19] is a large size iMSE designed to promote socialization skills, particularly among children with ASD.
The system stimulates the users to play together and collaborate with peers. A typical set-up involves one child with ASD and one neurotypical child.

SensoryPaint [27] is a smart environment that focuses on the expressivity of children with NDD, and is meant to be used by a single child or two children. Using connected objects or mid-air gestures, they can paint over a digital canvas, create drawings, and generate sounds.

The Magic Room presented in this paper is the latest version of the iMSE reported in [1] and [13], and results from a long technology re-engineering process and a countless number of design refinements and extensions. The first version was mainly intended for relaxation and free play of persons with NDD. The current version includes new activities conceived for mixed groups of children - with and without disability and have been designed in cooperation with a team of teachers, special education experts, and cognitive disability specialists. Also these activities are game-based but, compared with the ones of the first version of the Magic Room, they have a stronger educational flavor and offer more focused learning-oriented tasks. They involve a wider number of gesture-based and full-body interaction modes, richer multisensory effects, new digitally-enhanced physical objects, and a tablet-based application for caregivers, as discussed in the next section.

\section{THE MAGIC ROOM}

\section{Equipment and Technology}

The Magic Room requires an empty, white-painted room (sized a minimum-maximum of 2-3 meters in height, 3-5 meters in width, and 3-5 in length) and various technological devices that transform the space into a multisensory, interactive, and "magic" environment (Figure 1) by means of:

- $\quad$ visual contents projected on the walls and on the floor;

- ambient sound players

- smart physical objects (e.g., textured materials and stuffed toys)

- smart appliances such as bubbles makers, fragrance emitters, wireless portable smart lamps and fixed smart bulbs

- a Microsoft Kinect 2

- a mini PC that manages the generation and orchestration of the different stimuli.

Children interact with the Magic Room by manipulating objects and physical materials, or through movements in the space and mid-air gestures sensed by the Kinect. A novel and unique feature is a web application on a tablet (Figure 2) that enables caregivers to customize the experience in the Magic Room according to the needs of each children's group: selecting specific multimedia contents for each activity, setting the complexity level of the tasks, and organizing activities in automatically activated sequences. Using the same applications, caregivers can also "pause" or "repeat" 
the ongoing activity, "go back" to the previous one, "jump" to the next one.

This latter functionality allows them to control the activity flow at run-time( e.g., to dynamically to unlock situations when the children cannot complete the current task, or would benefit from repeating an activity).

The underlying technological system of the Magic Room is called "Magika"; it consists of a modular multi-layered
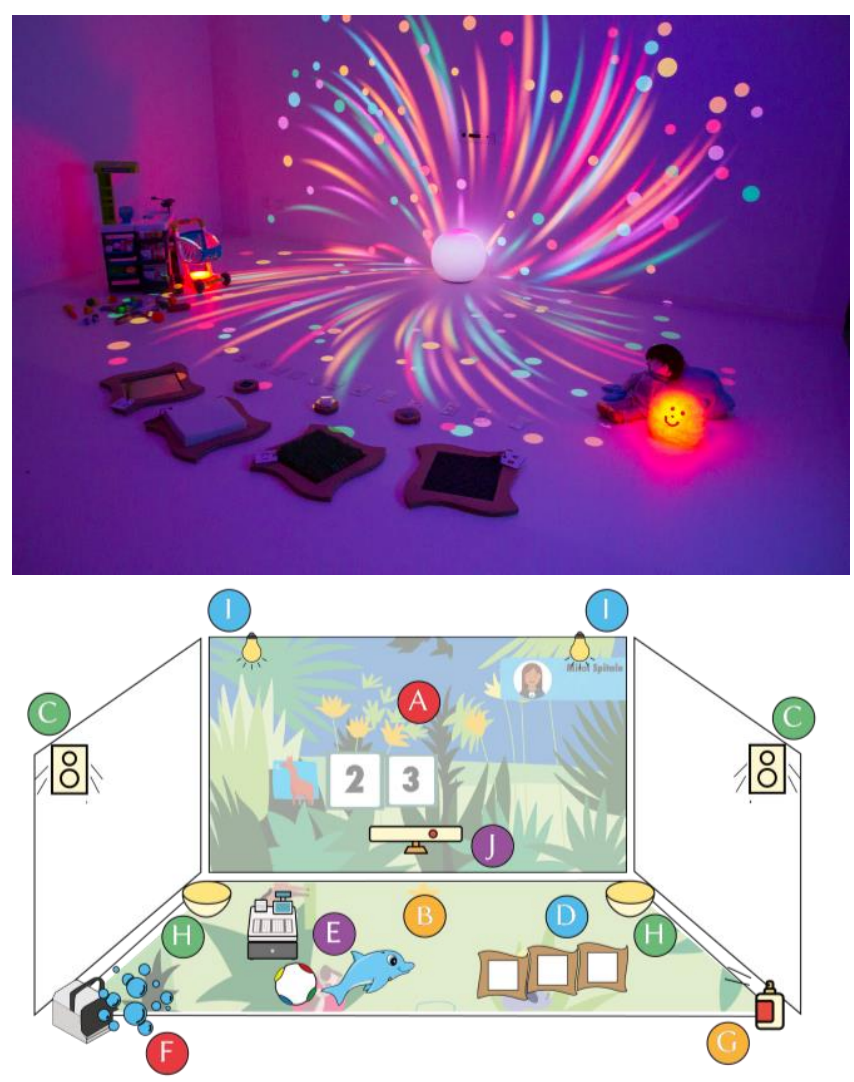

Figure 1. The Magic Room (top) and its main components (bottom): Front (A) and Floor (B) projection, audio system (C), Smart Materials (D), Smart Toys (E), Bubble Machine (F), Aroma Emitter (G), Lights (I, H), Kinect Sensor (J)

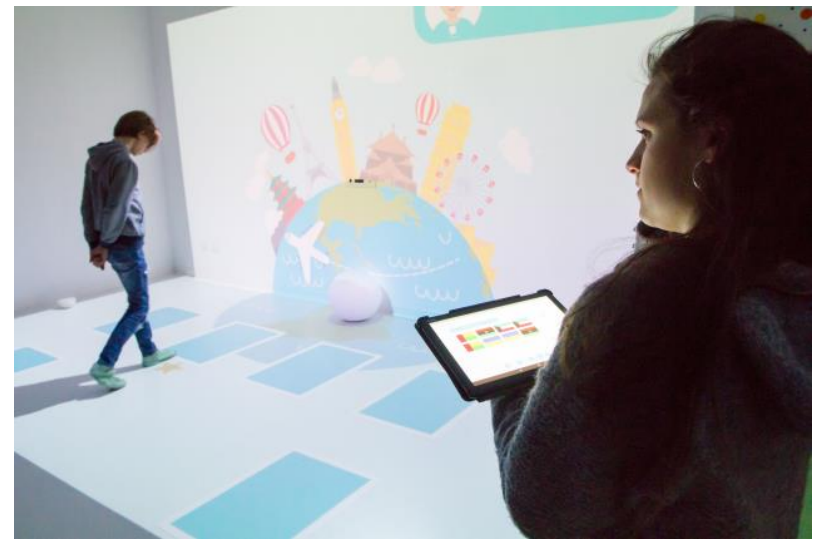

Figure 2. The Configuration and Control Application for caregivers architecture designed to be easily extendible with new software and hardware components to facilitate technology updates or implementation of new activities. The Web Service Layer - running on the tablet - manages the Configuration and Control Application for caregivers. The Activity Layer implements a set of "activity patterns", each of which manages the interaction, control and execution rules of a specific "type of activity" (described in the next section), and its instantiation - in terms of multimedia contents and specific UX features such as number of players, rewards, difficulty level - either setting a "default" configuration or a configuration defined by the caregivers themselves through the tablet app. The Middleware Layer comprises the modules to control the physical appliances, standardizing sensing and actuation capabilities for the upper levels, managing the communication between the hardware and software components and optimizing data exchange. New sensors and actuators can be replaced, added, or removed transparently, without affecting the upper level components of the Web Service Layer and the Activity Layer. The Physical Object Layer comprises the hardware components.

\section{Children's Activities}

The seven types of activities available in the current Magic Room are described below. Each of them can be customized in multiple ways to engage children in approximately 100 different activities. At the beginning of each activity, an animated virtual character appearing the front projection describes the activity goal and provide task instructions using speech and text.

\section{Classification Game}

A set of images and a set of labeled boxes are projected on the front wall. A child must "drag" (using a mid-air gesture) the images into the proper boxes. This activity is designed to improve working memory, attention, problem-solving and upper limb motor control.

\section{Association Game}

An environment (e.g., a wood, a music hall) is projected on the wall and a sequence of sounds is played. After each sound, some images are projected on the floor and the children are asked to identify the one associated to the sound (e.g., an animal noise or the music of an instrument) by moving over the selected image. This activity is designed to improve memory, attention, problem-solving and body control.

\section{Battleship Game}

This is a digital transposition of the classic Battleship board game adapted to the affordances of the Magic Room. Two projected grids conceal the positions of each team fleet. Players alternate turns "calling shots" at the other team's ships; but instead of using voice, children indicate the coordinates of the cell to hit by picking up two RFID tagged cardboards places in the room, one showing a number and the other showing a characters. To be recognized, tagged cards must be moved on top of a smart object (called "magic 
sphere") equipped with an embedded RFID reader and smart lights that provide a feedback when a card is read. This activity is designed to improve visuospatial skills, memory. problem-solving capability, and cooperation.

\section{Memory Game}

In this digital transposition of the classic Memory board game, children are asked to find a couple of identical digital cards among the ones projected (covered) on the floor. To "turn a covered card" they must move over it. This activity is designed to improve memory and visuospatial skills.

\section{Virtual Wardrobe}

The front projection shows a weather condition, and children should "choose" (moving on images projected on the floor) clothing items and accessories to dress up a virtual doll appropriately for the current weather. This activity is designed to improve autonomy, memory, attention, and executive functions.

\section{Grocery Game}

Front and floor projections create the effect of a virtual grocery shop. Children are asked to search and "buy" the items shown in a shopping list projected on the wall corner. The items are RFID-tagged plastic objects spread around the room. The chosen items must be brought to the child who plays the role of the cashier, who place them on the counter desk - another plastic object equipped with RFID reader, lights, and sound player. This activity is designed to improve autonomy, memory, attention, executive functions, problemsolving, visuospatial skills, language understanding, and fine motor skills.

\section{Immersive Game}

Children are immersed in a relaxing natural environment (e.g., the sea) created by front and wall video projections, while stimuli for the different senses are progressively generated: sound, soft ambient lights, and aromas. They can simply relax, or select some physical RFID tagged materials that are meaningful for the current environment and place them on the magic ball (like in the Battleship game) to smoothly change some video effects. This activity is designed to promote relaxation and well-being.

\section{Storytelling}

A voice tells a story that unfolds visually on front and wall projections. At some points of time, children are asked to perform gestures or movements, or to select a smart material in the room, consistently with the current situation of the story. This activity is designed to improve communication expression and interpretation skills.

\section{EMPIRICAL STUDIES: GENERAL CONSIDERATIONS}

\section{Location}

We performed two subsequent empirical studies in the two Magic Rooms installed in the primary schools (hosting children aged 5-11) located in a small town near Milan (Italy). According to the Italian regulation, all children must attend school up to $16 \mathrm{y} / \mathrm{o}$, and education must be inclusive; classes are typically composed of students with and without disability [5]. The groups recruited for our empirical studies were therefore "mixed", i.e., composed of children without and with disability, hereinafter referred to as "neurotypical" and "atypical" respectively. Atypical children had different forms of disability at different severity levels, including moderate to mild cognitive disability, specific learning impairments (e.g., Dyscalculia, Dysgraphia, Dyslexia), ASD, and Down Syndromes.

\section{Ethical issues}

Participant and their families were previously informed about the study. Informed consent was collected from parents or legal tutors. The informed consent forms included information about the study procedures, goals and data treatment. The Ethical Committees at our university and the two schools approved the study protocol. The issues of security, privacy, and confidentiality were addressed according to the guidelines provided by the European Data Protection Supervisor (EDPS).

\section{FIRST STUDY}

\section{Research Questions}

The focus of the first study was to investigate usability and organizational issues related to the Magic Room, specifically addressing the following research questions:

1. Is the Magic Room (MR) usable by mixed groups of children?

2. What is the appropriate size for the MR user group composed of children with mixed abilities and their teachers?

3. What is the appropriate duration of a session in the MR to prevent that children loose interest or become tired or unmanageable?

4. Which activities would children like the most?

\section{Participants}

We recruited 39 children from both schools: 21 males, 18 females, 22 neurotypical, and 17 atypical. In addition, 10 teachers and 5 researchers participated in the study. We divided participants into 3 groups (Table 1). Groups 1 and 2 were composed of two $4^{\text {th }}$ grade classes. For organizational reasons, group 3 was composed of atypical subjects only, aged 7 and 8 , and belonging to different classes.

\begin{tabular}{c|ccccc} 
Group & Tot & Male & Female & Atypical & Neurotyp. \\
\hline $\mathbf{1}$ & 19 & 10 & 9 & 8 & 11 \\
$\mathbf{2}$ & 17 & 9 & 8 & 6 & 11 \\
$\mathbf{3}$ & 3 & 2 & 1 & 3 & 0 \\
& Table 1. Groups Composition for Study $\mathbf{1}$
\end{tabular}

The study took place over two weeks; each group attended 2 sessions per week (4 sessions in total). All teachers participated in a "training workshop" in the Magic Room before the sessions started, had a demo of all available activities, and tried them. During each session, before entering the room the children waited in a dedicated adjacent 
space and prepared for the session, removing their shoes and wearing anti-slip socks. Then they were invited to enter the room with their two or three teachers, and to take a seat or to move freely. After a few minutes, each participant was required to sit quietly (on the benches or the floor) and the session started. Two researchers were present during all sessions: a member of the MR development team who managed the activities' flow interacting with the tablet application, and a psychologist from our lab who worked as observer. All groups experienced a predefined set of activities that were pre-configured at the basic level of complexity. The order and the type of the activities in the first three sessions were defined together with the teachers, taking into account children's cognitive level and group size. All sessions started with the Immersive Game. In the fourth session, the type and order of activities were chosen according to the children's preferences, obtained by vote (hands raising) after the end of the third session. Session organization is summarized in Table 2. At the end of the fourth session, children filled a paper-based questionnaire as discussed in the following section.

\begin{tabular}{|c|c|}
\hline GROUP & SESSION \\
\hline 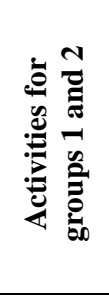 & $\begin{array}{l}\text { First session } \\
\text { \{Immersive Game; Memory; Classification Game; } \\
\text { Association Game } \\
\text { Second session } \\
\text { \{Immersive Game; Grocery Game; Virtual Wardrobe; Story } \\
\text { Third session } \\
\text { \{Immersive Game; Soft Material Story; Battleship } \\
\text { Fourth session } \\
\text { \{Immersive Game; Memory; Grocery Game; Battleship }\end{array}$ \\
\hline 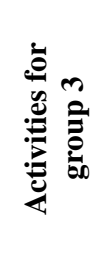 & $\begin{array}{l}\text { First session } \\
\text { \{Immersive Game; Virtual Wardrobe; Story }\} \\
\text { Second session } \\
\text { \{Immersive Game; Grocery Game; Association Game } \\
\text { Third session } \\
\text { \{Immersive Game; Soft Material; Classification Game } \\
\text { Fourth session } \\
\text { \{Immersive Game; Virtual Wardrobe; Grocery Game }\end{array}$ \\
\hline
\end{tabular}

Table 2. Study 1: Activity flows for each session and group

\section{Data gathering}

Observations were annotated manually by the observer to record the relevant phenomena in children's behavior. Final feedback was collected from children using a four-values Smileyometer (Figure 3), asking them to express their degree of likeability for each activity as well for whole experience in the Magic Room ("global likeability"). At the end of the study, we organized a focus group with the 10 teachers involved in the study.
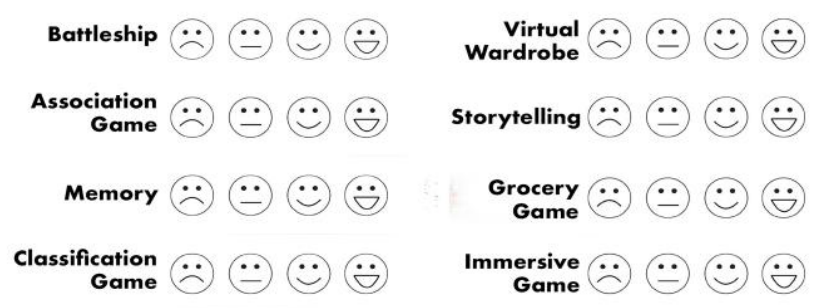

Figure 3. Study 1: Children's Questionnaires on Likeability

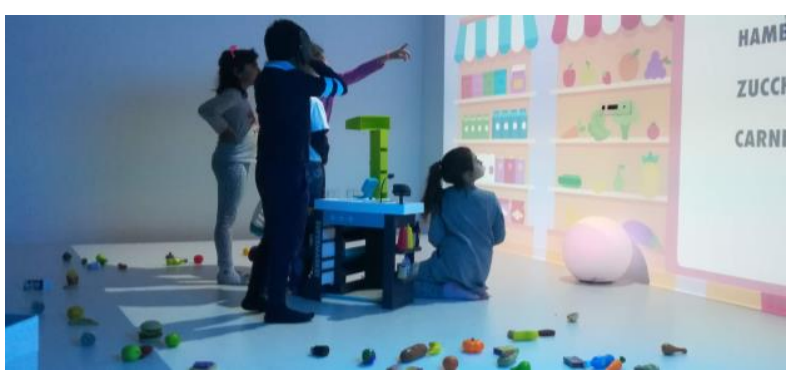

Figure 4. Children playing Grocery Game (top) and Immersive Game (bottom).

\section{FIRST STUDY: RESULTS AND DISCUSSION}

Is the Magic Room (MR) usable by mixed groups of children? Our data suggest that the MR is suitable for both mixed groups of children as well as small groups composed of atypical children only. All participants seemed to be engaged during all the activities - no signal of boredom or intolerance was observed even among children not directly involved in the execution of a task, and they all expressed positive feedbacks in the questionnaire. During the final focus group teachers confirmed these observations.

What is the appropriate size for the MR user group composed of children with mixed abilities and their teachers?

The MR seems to be usable both with large mixed groups and with small groups: group 1 had 19 children and group 2 had 17 children; group 3 had 3 atypical children. For actionbased activities, we suggest an ideal number of up to 10 children, while relaxation activities seem to be appropriate for up to 15 children. In general, some precautions must be taken with large groups $(>15)$ : at least three teachers should be present, to play as facilitator during task execution, as manager of turn-taking during the activities, and as moderator for the children not interacting in person with the Magic Room; in cooperative activities, children's roles should be clearly defined since the very beginning, e.g., defining who is in charge of moving/gesturing, searching or passing the physical materials, and helping another child.

What is the appropriate duration of a session in the MR to prevent that children loose interest or become tired or unmanageable?

Each session was initially planned to last from 40 to 60 minutes. The longest permanence in the MR was 58 minutes, while the shortest was 36 minutes. The suggested duration could be around 45 minutes per session. Our observations also suggest that this period can be extended using more relaxing activities such as the Immersion game and Storytelling.

Which activities would children like the most?

The main results of the analysis of quantitative data from the children's likability questionnaires are described in Table 3. All activities scores ranged between 3 and 4 . Association $(\boldsymbol{\mu}$ $=3,06, \boldsymbol{\sigma}=, 864)$ and Classification $(\boldsymbol{\mu}=3,03, \boldsymbol{\sigma}=1,045)$ games are those with the lowest score. 


\begin{tabular}{c|cccc}
\multicolumn{2}{c}{} & $\boldsymbol{\mu}$ & Median & $\boldsymbol{\sigma}$ \\
\multicolumn{2}{c}{ Overall experience } & 3,70 & 4 &, 651 \\
\hline & Association & 3,06 & 3 &, 864 \\
& Classification & 3,03 & 3 & 1,045 \\
& Battleship & 3,36 & 4 &, 859 \\
$:$ & Memory & 3,36 & 4 &, 859 \\
& Wardrobe & 3,21 & 4 &, 992 \\
& Grocery & 3,70 & 4 &, 728 \\
& Immersive & 3,42 & 4 &, 902 \\
& Story & 3,36 & 4 &, 783
\end{tabular}

Table 3. Activities scores from children's questionnaires

Probably because, compared to other games, these are less game-based and competitive activities and more logical and reasoning activities. Therefore may be less appealing to children. On the other hand, Grocery $(\mu=3,70, \sigma=, 728)$ and Immersive $(\mu=3,42, \sigma=, 902)$ games are the ones with the highest score. Both activities are those that best express the multisensoriality and the immersivity of the MR. The Grocery game provides a continuous tangible interaction through objects' manipulation and interaction with projections, sound and visual feedback, while the Immersive game was specifically designed with the aim of immerging subjects through a progressive exposure to environmental stimuli. These aspects seem to be very appealing to children.

Observers' notes confirmed these results, highlighting a higher degree of children's engagement in Grocery and Immersive games. Accordingly, to teachers' impressions, Memory and Immersive games were the most appreciated ones. In addition, teachers suggested that Immersive games could be used as the "closing experience", as these activities help to mitigate children's excitement that is often high at the end of a session in the Magic Room. According to the comments of both neurotypical and atypical children, all activities seem to be liked: "They are all fun games"; "I don't have a favourite game: I like them all"; "I never get tired in the Magic Room!"; " None of the games bored me. They are all super fun"; "I liked staying in the room very much, can I suggest a new activity?".

A one-way ANOVA was computed to validate the effect on likeability of both the overall experience and each single activity: Overall experience $[\mathrm{F}(2,27)=21,41, \mathrm{p}=0.000]$, Battleship $[\mathrm{F}(2,27)=5,59, \mathrm{p}=0.009]$, Association $[\mathrm{F}(2,27)$ $=4,65, \mathrm{p}=0.017]$, Memory $[\mathrm{F}(2,27)=5,59, \mathrm{p}=0.009$, Wardrobe $[\mathrm{F}(2,27)=2,88, \mathrm{p}=0.007]$ and Grocery $[\mathrm{F}(2,27)=12,31, \mathrm{p}=0.000]$. Post-hoc comparisons using the Bonferroni test confirmed the significant effect $(\mathrm{p}<.05)$ for six conditions ("Overall experience", "Battleship", "Association", "Memory", "Wardrobe" and "Grocery") but not on the "Classification", "Immersive" and "Story".

Overall, our analysis suggests that the overall experience in the Magic Room and some specific activities - "Battleship", "Association", "Memory", "Wardrobe" and "Grocery" affect children's likability.

\section{SECOND STUDY}

\section{Research Questions}

The goal of the second study was to explore the impact of the Magic Room on both atypical and neurotypical children, focusing on the following Research Questions:

1. Do the experiences in the Magic Room affect children's well-being?

2. How does a mixed group of neurotypical and atypical children behave in the Magic Room?

\section{Research Variables}

Well-Being

Well-being refers to optimal psychological functioning, and it is a broad concept and a sensitive variable to measure. We did not focus not on children's general well-being, but on a specific dimension: "well-being at school", which considers how well children feel in the school environment.

\section{Behavior}

Behavior is a complex dimension to study, and in the current literature there are various theories and measurement tools [3][8[16][25][29].

A number of studies [15][17][23][30][33] explore positive and negative effects of (i)MSEs on the behavior of children

\begin{tabular}{l|l} 
BIMENSION & \multicolumn{1}{|c}{ BEHAVIORAL SIGNALS } \\
\hline $\begin{array}{l}\text { Positive } \\
\text { laugh, positive comments, exult, jump, } \\
\text { quietness, cheer } \\
\text { Negative } \\
\text { negative comments, request to end the } \\
\text { activity, loss of attention, facial sign of } \\
\text { frustration, rage, fear, fatigue, room } \\
\text { abandonment }\end{array}$ \\
$\begin{array}{ll}\text { Positive } \\
\text { congratulate with the companions, invite a } \\
\text { companion to participate, respect the turn, } \\
\text { self-organization, share, help, ask for peers } \\
\text { help, respect of rules, effusion, spontaneous } \\
\text { help } \\
\text { Negative } \\
\text { Bullying, rude gesture, ignore peers, refuse to } \\
\text { do what asked from peers, isolation, } \\
\text { aggressive actions towards peers, aggressive } \\
\text { actions towards the room, not respect of the } \\
\text { rules }\end{array}$ \\
\hline
\end{tabular}

Table 4. Study 2: Study 2: Behavioral Variables 
with autism or other forms of disability. To the best of our knowledge, no prior work investigates the behavioral effects of iMSEs on mixed groups of atypical and neurotypical children interacting.together in an iMSE. For the purpose of our study, we designed and used a tailored sets of behavioral dimensions (Table 4) associated to positive and negative behavioral signals. The items in our scale are inspired to practices and taxonomies reported in the literature and concerning both neurotypical and atypical subjects [4] [11][14][26][28].

\section{Data gathering}

\section{Well-being questionnaires}

Our well-being measurement tools was QBS 8-13 Questionnaire on Well-Being at School [18][31] - a validated standardized paper-based questionnaire widely used in Italy to monitor well-being in educational contexts for children aged 8 to 13 . QBS consists of three different questionnaires: one for the child, one for the parents, and one for the teachers. All participants filled the QBS questionnaire twice; before the beginning of the study (Baseline condition) and after the end of the study (Post condition). QBS Questionnaires were delivered and returned in blank envelopes to ensure privacy.

\section{Behavioral Observations}

To facilitate the recording of observations on children's behavior during the Magic Room sessions, we created a simple tablet-based web application called B.O.A (Behavior Observation Web Application). When the observer noticed a relevant behavior for one or more children, he/she could simply touch the screen twice: for the corresponding behavioral signal and for the number the number of children who manifested that behavior. The application automatically stored the selections associated to a timestamp. Observers were psychologists outside our research group who were recruited for the study. Before the study, they received a training on the Magic Room and B.O.A., and practiced on behavioral observations, achieving an inter-rater reliability of $95 \%$.

\begin{tabular}{c|ccccc} 
GROUP & Total & Male & Female & Atypical & Normotyped \\
\hline $\mathbf{1}$ & 19 & 10 & 9 & 8 & 11 \\
$\mathbf{2}$ & 17 & 9 & 8 & 6 & 11 \\
$\mathbf{3}$ & 11 & 6 & 5 & 4 & 7 \\
$\mathbf{4}$ & 5 & 2 & 3 & 2 & 3 \\
$\mathbf{5}$ & 2 & 2 & 0 & 2 & 0 \\
$\mathbf{6}$ & 5 & 2 & 3 & 4 & 1 \\
$\mathbf{7}$ & 9 & 6 & 3 & 5 & 4
\end{tabular}

Table 5. Study 2: Children's Groups Composition.

\section{Participants}

The second study involved 68 children - 46 atypical and 22 neurotypical children, aged 7-8 y/o (mean age $=7$, s.d.=1.3), 12 teachers and 7 observers. Participants were organized into 7 mixed groups as illustrated in Table 5.

\section{Procedure}

The study lasted for three weeks, during the last school period (from approximately the mid of May to the beginning of June). Each group participated in one session every 5 days, for a total of 4 sessions. Each session lasted from 45 to 60 minutes. Three researchers and three teachers were present. in each session. Two researchers filled the B.O.A. for neurotypical and atypical subjects respectively; the third researcher managed the Control and Configuration App.

The general procedure was similar to study 1 . In each session each groups performed a predefined set of activities at a basic level of complexity. Activities were defined together with the teachers according to the subjects' cognitive level and the size of the group. We assigned activities that required a high cognitive level to groups 1, 2, 6 and 7, while group 3,4 and 5 performed less cognitively demanding activities. The order of the activities was counterbalanced to ensure correct randomization.

\begin{tabular}{|c|c|}
\hline GROUPS & ACTIVITIES PER SESSION \\
\hline 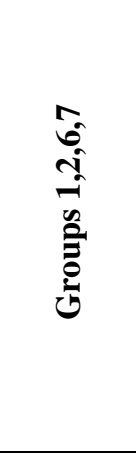 & $\begin{array}{l}\text { First session } \\
\text { \{Memory; Classification Game; Association Game; } \\
\text { Immersive Game } \\
\text { Second session } \\
\text { \{Classification Game; Immersive; Memory; } \\
\text { Association Game } \\
\text { Third session } \\
\{\text { Immersive Game; Association Game; Immersive } \\
\text { Game } \\
\text { Fourth session } \\
\text { \{Association Game; Memory; Immersive Game } \\
\text { Classification Game }\end{array}$ \\
\hline 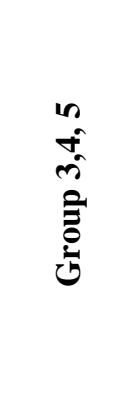 & $\begin{array}{l}\text { First session } \\
\{\text { Immersive Game; Story; Immersive Game }\} \\
\text { Second session } \\
\{\text { Immersive Game; Grocery Game; Immersive } \\
\text { Game }\} \\
\text { Third session } \\
\{\text { Immersive Game; Association game; Immersive } \\
\text { Game }\} \\
\text { Fourth session } \\
\{\text { Immersive Game; Virtual Wardrobe; Immersive } \\
\text { Game }\}\end{array}$ \\
\hline
\end{tabular}

Table 6. Study 2: Activities for each sessions and group SECOND STUDY: RESULTS AND DISCUSSION

\section{Well-being}

For organizational reasons, we could collect the complete well-being data about 36 children (19 males and 17 females, 22 neurotypical and 14 atypical) extracted from 216 questionnaires. For each subject, we had 3 questionnaires for the before-study baseline condition - one from the child, one from the parents, and one from the teachers, and 3 questionnaires for the post-study control condition. Questionnaire results were analyzed and scored according to the standardized QBS procedure that interprets the 
questionnaire values in terms of six different indicators. The principal one is the General Score that summarises an individual's general well-being in the school context. The five additional indicators (or "subscales") measure more specific aspects related to well-being: Satisfaction, Relationship with Teachers, Relationship with Peers, Emotional Attitude (the psychological tendency to evaluate a particular entity or phenomenal positively or negatively), Self-efficacy (an individual's belief in his or her capacity to execute behaviors necessary to produce specific performance accomplishments).

Each indicator is measured on a five values qualitative scale mapped to a numerical scale: seriously poor $=1$; poor $=2$; medium $=3$; normal $=4$; above normal $=5$. For each indicator and each child, we considered the measures in the two conditions (baseline and post) as well as $\Delta$ values, i.e., the differences between the baseline and post conditions values. A positive (resp. negative) $\Delta$ indicates an improvement (resp. aweakening) of well-being, either general or for a specific dimension. The rest of this section presents and discussed the results emerging from children's questionnaires, since finding from parents' and teachers' questionnaires before and after the study.

\section{General score}

The main results for the General Score (baseline: $\mu=3,75, \sigma$ $=0,91$; post: $\mu=3,97, \sigma=0,70)$ are illustrated in Figure 5 that plots aggregated data distinguishing between neurotypical and atypical subjects and between baseline and post conditions.

Results highlight that:

- In the Baseline condition, among neurotypical children: 5 subjects had an "above normal" value, 13 "normal" value, 2 "medium" value, and 2 "below normal" value. According to the literature [18], these findings indicate that our neurotypical participants were a generally healthy population in terms of well-being. For atypical children, 9 subjects children had "normal" value, 3 "medium" value, 4 "poor" value, and 1 "seriously poor" value. For this population, well-being values were more polarized towards the lowest levels in the scale, a result which is intuitive (the impairments derived from disability affect the quality of psychological functioning) and coherent with the exiting literature [8].

- In the Post condition, for the neurotypical population, the values changes only slightly, with 4 children achieving "above normal" value, 15 a "normal" value, and 3 "medium" value. Well-being improvement seems to occur among the atypical population: no child scored "seriously poor" as it happened in the baseline condition; 12 children achieved a "normal" value, 3 a "medium" value, and 2 a "poor" value.

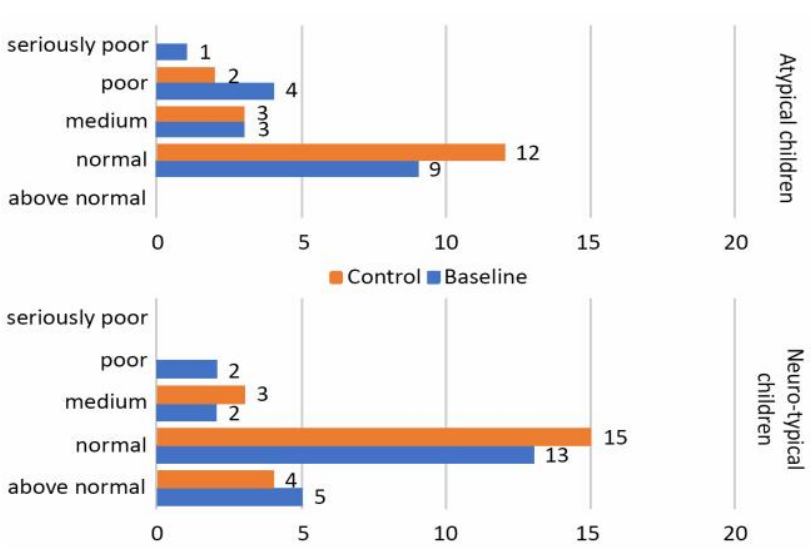

Figure 5. Children's Well-being General Scores for Baseline and Post.

- The General Score $\Delta$ was positive and statically significant, both for the neurotypical population and the atypical population. Moreover, General Score $\Delta$ of atypical subjects $(0,89 ; \mathrm{t}(17)=0,0002, \mathrm{p}<.002)$ was better than General Score $\Delta$ of neurotypical subjects $(0,14 ; \mathrm{t}(22)=0,0001, \mathrm{p}<.002)$.

\begin{tabular}{c|cccccc} 
& & $\mathrm{S}$ & $\mathrm{T}$ & $\mathrm{P}$ & $\mathrm{E}$ & $\mathrm{Se}$ \\
\hline \multirow{\sigma}{*}{} & $\mu$ & 3,89 & 4,03 & 4,03 & 3,42 & 3,78 \\
& $\sigma$ & 0,95 & 0,88 & 1,06 & 1,05 & 0,76 \\
$\underset{\rightleftharpoons}{\rightleftharpoons}$ & $\mu$ & 3,83 & 4,03 & 3,97 & 3,78 & 3,61 \\
& $\sigma$ & 0,85 & 0,88 & 1,00 & 0,76 & 0,93
\end{tabular}

Table 7. QBS mean and SD for Satisfaction (S), Relationship with Teachers (T), Relationship with Peers (P), Emotional attitude (E), and Self-efficacy (Se)

Sub-scales

Table 7 shows the main results for the five QBS subscales, and highlights that:

- For atypical subjects, scores had positive $\Delta$ for 4 indicators out of 5: Relationship with Peers was 0,57 ( $\mathrm{t}(17)=0,0012$, $\mathrm{p}<.002) ; \quad$ Relationship with Teachers was 0,5, $(\mathrm{t}(17)=0,0008, \mathrm{p}<.002)$, and Emotional attitude was 0,71 $(\mathrm{t}(17)=0,0001, \mathrm{p}<.002)$;

- For neurotypical subjects, only Emotional attitude had a positive $\Delta(0,23 ; \mathrm{t}(22)=0,0009, \mathrm{p}<.002)$, while the other indicators have a negative $\Delta$.

Final remarks

Figure 7 plots aggregated data for all well-being indicators, distinguishing between neurotypical and atypical subjects and between baseline and post conditions.

Overall, the values of atypical children seem to have a better trend than those of neurotypical subjects. According to our findings, even a relative short experience in the Magic Room might positively affect perceived well-being in the school integration environment, especially for atypical children. 


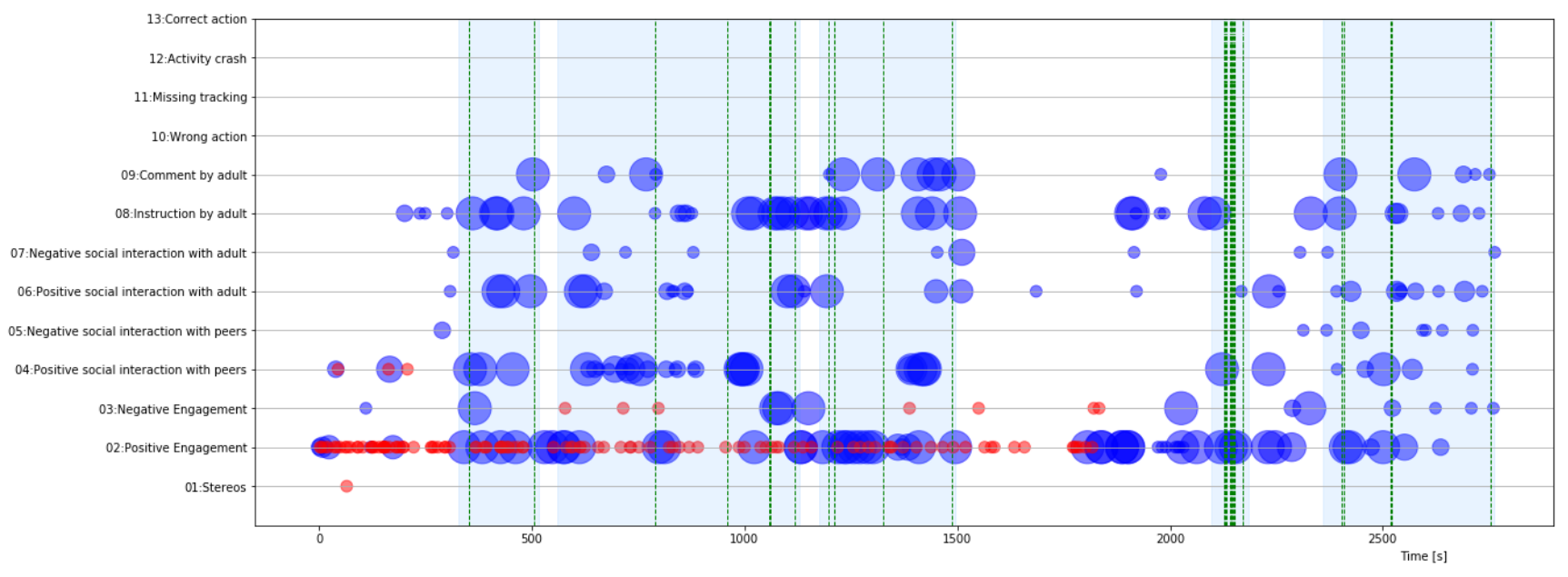

Figure 6: Study 2: Visualization of behavioural data in session 1 for atypical children (red dots) and typical children (blue dots) in Group 1.

These effects seem to be stronger for some specific wellbeing indicators such as Emotional Attitude and Social Interaction (with peers and with adults) that are related to psychological functioning dimensions that are particularly critical for this population.

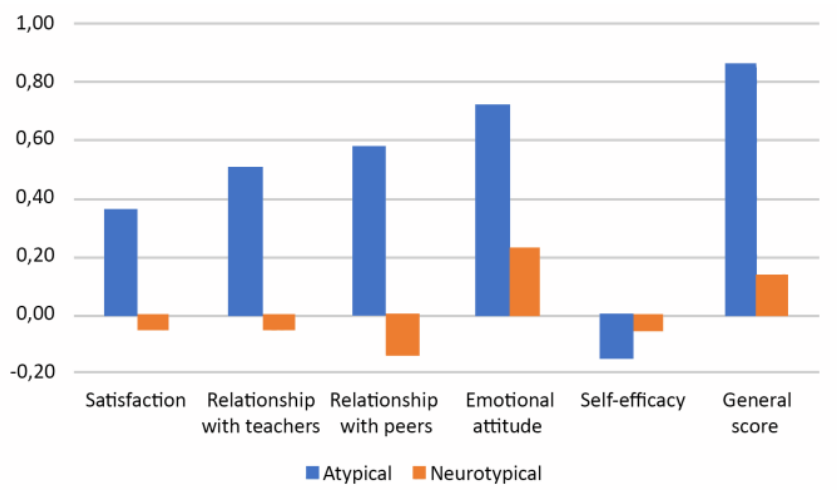

Figure 7. Well-being values for all indicators

\section{Behavior}

Since we had two different observers for neurotypical and atypical children, the information collected with the B.O.A application - behavioral signal observed at a given point of time and number of children manifesting that behavior could be clustered by population type. Triangulating these results with the log data of the Magic Room, we could also associate behavioral information with the type of activity and the specific group of participants. The resulting data was represented in a series of 3 scatter plots for each session: two for each type of population, and one aggregating the results of typical and atypical children. In the resulting 96 visualizations, for each behavioral signal we used red dots for atypical children and blue dots for typical subjects, and dot size to suggest the dimension of the group of children who manifested that behavior at a given point of time. The rest of this section discusses the main results emerging for the analysis of these visualizations, which, for lack of space and for readability purpose, are all reported as an Annex to this paper. One example is shown in Figure 6.

In general, our visualizations highlight that all children manifested much more positive than negative behavior (the lines corresponding to negative signals are empty most of the time). They seemed to adopt a generally positive attitude when they were in the Magic Room.

Concerning engagement, the distribution of behavioural signals of this type was rather regular along the time, regardless of what was happening in the Magic Room, and there were no relevant differences also between different sessions. These findings seem to indicate that children's engagement might be not influenced by the contingent status of the experience in the Magic Room, i.e., "activity running" or "idle". During activity execution, engagement is probably motivated by the nature of action-driven games, and the pleasurable effects of the Immersion and Storytelling games. During the "idle" moments of transition from an activity to the next one, engagement might be due to the soft stimulation, the immersive effects, and the aesthetic quality of the multimedia contents automatically generated by the space with projections of beautiful rhythmic animations on the wall and the floor synchronized with pleasant music and dynamic light effects.

Focusing on the different types of children's population, some differences emerged between atypical and neurotypical subjects. In general, the range and variety of behavioral signals was wider in neurotypical children w.r.t. to atypical ones, but the variety of positive behaviors for the latter slightly increased along the time. In addition, during the first (and, sometimes, the second) session, negative engagement signals such as fear, loss of attention, and frustration were observed for few atypical children. These findings are consistent with the characteristics of this population: any 
novel situation is intrinsically complex for them to manage and creates stress and cognitive load [2][7], particularly if it offers new and rich stimuli. It is important to pinpoint that in the Magic Room these behaviors tended to disappear with time, even when new activities were proposed.

\section{LIMITATIONS OF THE STUDIES}

In our empirical research we had to face many challenging factors that are typical of "working in the wild". Even though the results are promising, both studies have several limitations, mostly concerning the heterogeneity of the groups size and their members, and the duration of the study. A number of problems were unavoidable and originated from the context in which the Magic Rooms are installed, i.e., public schools that have temporal and educational constraints rigidly regulated at national level. Both schools were very collaborative, but the design and execution of the empirical research were limited by organizational and educational issues often conflicting with our scientific needs; for example, we could not manage the recruitment of participants directly, establish the composition of children groups, prolong the study duration and schedule the sessions in the way we desired. Both studies were conducted for a relatively short time, which weakens the statistical validity. We cannot exclude, for example, that likeability and some positive behaviours measured during the sessions in the Magic Room are not ascribed to novelty effects. Nor we know if the latter could be generalized to other moments of children's every-day life, at school or in other contexts. At the end, the execution of the two studies was the best possible compromise between researchers' requirements and schools' rules and plans.

Concerning specific research variables, those related to children's well-being are particularly complex to measure. They are influenced by multiple aspects [22] which affect intensity, duration, and polarization [31]. Confounding variables that we could not control are related to subjective and contingent factors such as physiological and psychological state, or contextual aspects such as family or social conditions [10]. Still, it is important to remind that our well-being measurement instrument investigates well-being related to the school experience only. According to the relevant literature, QBS questionnaires and associated data analysis procedures have been designed and validated taking into account the main potentially influencing factors related to what happens outside the school context, e.g., at home.

\section{CONCLUSIONS}

The paper has presented an interactive Multi-Sensory Environments (iMSE) called Magic Room and explored its adoption in real educational settings - two local primary schools, and for mixed groups of neurotypical and atypical children. The value of our work is related to a number of dimensions.

Technology. We mastered the complexity of integrating multiple connected devices and software/hardware components in a robust and performant way.
User Experience. Our Magic Room provides a wealth of interactive multisensory activities for children that seems to keep them engaged not just sporadically but for many sessions and for long periods. At the time of writing this paper, the two Magic Rooms have been used for over 8 months on a regular base. The teachers involved in our studies keep bringing their children in the Magic Rooms ones or twices a week, autonomously and without experiencing any technical problem. Our hope is that these enthusiastic "early adopters" will stimulate other teachers to try our technology, creating local communities of educators that will contribute to identify innovative technology-empowered inclusive education processes at school.

Empirical results on inclusive education. Despite some limitations, our field studies are unique in the iMSE arena for their duration as well as number and typology of the subjects involved. They offer a preliminary evidence of the potential of iMSEs for inclusive education, suggesting that these systems could be effective for children with and without disability to improve behavior, socialization skills, and motivation. Our studies also highlight the many socioorganizational challenges emerging when investigating complex technology in real educational contexts, and shed a light on how the technology adoption process can be managed.

An issue for lack of space we have not discussed in depth in the paper is how to empower teachers, so that they can become not only autonomous users of iMSEs, but also creators of new ways of exploiting them for educational purposes. In our opinion, this empowering process can be helped in several ways: involving teachers in design work (as we did as much as possible for both the activities inside the Magic Room and the empirical studies); offering them a simple yet powerful configuration and control tool for the children experiences inside the iMSE, like the tablet application available in the Magic Room; investing time and human resources in training teachers, as we did in our project.

\section{ACKNOWLEDGMENTS}

The authors are grateful to the children, the parents, the teachers and the managers at the two schools in Cornaredo (Milan) where the Magic Room is installed. This work has been partially supported by Polisocial - The Social Responsibility Program of Politecnico di Milano.

\section{REFERENCES}

[1] Agosta, G., Borghese, L., Brandolese, C., Clasadonte, F., Fornaciari, W., Garzotto, F., ... \& Valla, M. (2015, August). Playful Supervised Smart Spaces (P3S)--A Framework for Designing, Implementing and Deploying Multisensory Play Experiences for Children with Special Needs. In 2015 Euromicro Conference on Digital System Design (pp. 158-164). IEEE. 
[2] Bacon, A. L., Fein, D., Morris, R., Waterhouse, L., \& Allen, D. (1998). The responses of autistic children to the distress of others. Journal of autism and developmental disorders, 28(2), 129-142.

[3] Baldwin, A. L. (1955). Behavior and development in childhood.

[4] Beccaluva, E. A., Bonarini, A., Cerabolini, R., Clasadonte, F., Garzotto, F., Gelsomini, M., ... \& Viola, L. (2017). Exploring engagement with robots among persons with neurodevelopmental disorders. In 2017 26th IEEE International Symposium on Robot and Human Interactive Communication (RO-MAN) (pp. 903-909). IEEE.

[5] Benigno, V., Epifania, O., Fante, C., Ravicchio, F., \& Trentin, G. Classi ibride per promuovere relazioni e apprendimenti.

[6] Brule, E., Bailly, G., Brock, A., Valentin, F., Denis, G., \& Jouffrais, C. (2016, May). MapSense: multisensory interactive maps for children living with visual impairments. In Proceedings of the 2016 CHI Conference on Human Factors in Computing Systems (pp. 445-457). ACM.

[7] Cadman, D., Boyle, M., Szatmari, P., \& Offord, D. R. (1987). Chronic illness, disability, and mental and social well-being: findings of the Ontario Child Health Study. Pediatrics, 79(5), 805-813.

[8] Carter, E. W., Hughes, C., Guth, C. B., \& Copeland, S. R. (2005). Factors influencing social interaction among high school students with intellectual disabilities and their general education peers. American Journal on Mental Retardation, 110(5), 366-377.

[9] Colby, K. M. (1973). The rationale for computerbased treatment of language difficulties in nonspeaking autistic children. Journal of Autism and Childhood Schizophrenia, 3(3), 254-260.

[10] Diener, E., Diener, M., \& Diener, C. (2009). Factors predicting the subjective well-being of nations. In Culture and well-being (pp. 43-70). Springer, Dordrecht.

[11] Eyberg, S. M., \& Ross, A. W. (1978). Assessment of child behavior problems: The validation of a new inventory. Journal of Clinical Child \& Adolescent Psychology, 7(2), 113-116.

[12] Foglia, L., \& Wilson, R. A. (2013). Embodied cognition. Wiley Interdisciplinary Reviews: Cognitive Science, 4(3), 319-325.

[13] Garzotto, F., \& Gelsomini, M. (2018). Magic Room: A Smart Space for Children with Neurodevelopmental Disorder. IEEE Pervasive Computing, 17(1), 38-48.

[14] Ladd, G. W., \& Profilet, S. M. (1996). The Child Behavior Scale: A teacher-report measure of young children's aggressive, withdrawn, and prosocial behaviors. Developmental psychology, 32(6), 1008 .
[15] Lancioni, G. E., Cuvo, A. J., \& O'reilly, M. F. (2002). Snoezelen: an overview of research with people with developmental disabilities and dementia. Disability and rehabilitation, 24(4), 175184.

[16] Liu, J. (2004). Childhood externalizing behavior: Theory and implications. Journal of child and adolescent psychiatric nursing, 17(3), 93-103.

[17] Lotan, M., \& Shapiro, M. (2005). Management of young children with Rett disorder in the controlled multi-sensory (Snoezelen) environment. Brain and Development, 27, S88-S94.

[18] Marzocchi, G. M., \& Tobia, V. (2015). QBS 8-13: Questionari per la valutazione del benessere scolastico e identificazione dei fattori di rischio. Edizioni Centro Studi Erickson.

[19] Mora-Guiard, J., Crowell, C., Pares, N., \& Heaton, P. (2017). Sparking social initiation behaviors in children with Autism through full-body Interaction. International Journal of ChildComputer Interaction, 11, 62-71.

[20] Pagliano, P. (2017). Multisensory environments. David Fulton Publishers.

[21] Panyan, M. V. (1984). Computer technology for autistic students. Journal of Autism and Developmental Disorders, 14(4), 375-382.

[22] Parasuraman, S., Purohit, Y. S., Godshalk, V. M., \& Beutell, N. J. (1996). Work and family variables, entrepreneurial career success, and psychological well-being. Journal of vocational behavior, 48(3), 275-300.

[23] Parés, N., Carreras, A., Durany, J., Ferrer, J., Freixa, P., Gómez, D., ... \& Sanjurjo, À. (2005, June). Promotion of creative activity in children with severe autism through visuals in an interactive multisensory environment. In Proceedings of the 2005 conference on Interaction design and children (pp. 110-116). ACM.

[24] Pares, N., Masri, P., Van Wolferen, G., \& Creed, C. (2005). Achieving dialogue with children with severe autism in an adaptive multisensory interaction: the" MEDIATE" project. IEEE Transactions on Visualization and Computer Graphics, 11(6), 734-743.

[25] Patterson, G. R., Littman, R. A., \& Bricker, W. (1967). Assertive behavior in children: A step toward a theory of aggression. Monographs of the society for research in child development, 32(5), iii43.

[26] Reynolds, C. R. (2010). Behavior assessment system for children. The Corsini encyclopedia of psychology, 1-2.

[27] Ringland, K. E., Zalapa, R., Neal, M., Escobedo, L., Tentori, M., \& Hayes, G. R. (2014, September). SensoryPaint: a multimodal sensory intervention for children with neurodevelopmental disorders. In Proceedings of the 2014 ACM International Joint 
Conference on Pervasive and Ubiquitous Computing (pp. 873-884). ACM.

[28] Schopler, E., Reichler, R. J., DeVellis, R. F., \& Daly, K. (1980). Toward objective classification of childhood autism: Childhood Autism Rating Scale (CARS). Journal of autism and developmental disorders, 10(1), 91-103.

[29] Selleri, P., Gaffuri, P., \& Mazzoni, E. (2006). Oltre Moreno: l'uso delle reti sociali come strumento di socializzazione e benessere in classe. Psicologia della salute.

[30] Stadele, N. D., \& Malaney, L. A. (2001). The effects of a multisensory environment on negative behavior and functional performance on individuals with autism. Journal of undergraduate research. University of Winsconsin-La Crosse.

[31] Tobia, V., Greco, A., Steca, P., \& Marzocchi, G. M. (2019). Children's wellbeing at school: A multidimensional and multi-informant approach. Journal of Happiness Studies, 20(3), 841-861.

[32] Tomchek, S. D., \& Dunn, W. (2007). Sensory processing in children with and without autism: a comparative study using the short sensory profile. American Journal of occupational therapy, 61(2), 190-200.

[33] Tunson, J. N., \& Candler, C. (2010). Behavioral states of children with severe disabilities in the multisensory environment. Physical \& occupational therapy in pediatrics, 30(2), 101-110.

[34] Tüzün, H., Yılmaz-Soylu, M., Karakuş, T., İnal, Y., \& Kizlkaya, G. (2009). The effects of computer games on primary school students' achievement and motivation in geography learning. Computers \& Education, 52(1), 68-77.

[35] Wilson, M. (2002). Six views of embodied cognition. Psychonomic bulletin \& review, 9(4), 625-636. 